\title{
A new evidence of therapeutic effects of Mycobacterium bovis BCG vaccine to experimental autoimmune encephalomyelitis (EAE): BCG-mediated suppression of Th17 response in EAE mice induced using an adjuvant without mycobacterial antigen.
}

\author{
Goro Matsuzaki $^{1}$, Naoko Teruya ${ }^{1}$, Hideyasu Kiyohara ${ }^{2}$, Keiko Arai $^{2}$, Yukihiro Shibuya $^{2}$, \\ Yasushi Chuma ${ }^{2}$, and Kazuhiro Matsuo ${ }^{2}$ \\ ${ }^{1}$ University of the Ryukyus \\ ${ }^{2}$ Japan BCG Laboratory Kiyose Research Center
}

August 23, 2020

\begin{abstract}
Multiple sclerosis (MS) is an autoimmune disease mediated by myelin autoantigen-specific T cells. Experimental autoimmune encephalomyelitis (EAE) induced by immunization of mice with a myelin oligodendrocyte glycoprotein (MOG) peptide emulsified in killed Mycobacterium tuberculosis-containing complete Freund's adjuvant (CFA-EAE) is frequently used as a model of MS. Mycobacterium bovis BCG, a vaccine strain with various biological response modifier activity, has been reported to ameliorate clinical symptoms of the CFA-EAE although precise mechanism has not yet been documented. Since the CFA-EAE uses adjuvant with mycobacterial antigens, it is possible that mycobacterial antigen-specific T cells induced by CFA and those by therapeutic BCG inoculation recognize same antigens, and the cross-reactivity modulate the EAE. To exclude the influence of the cross-reactivity, we established a modified murine EAE model (CWS-EAE) which does not induce mycobacterial antigen-specific T cells. Inoculation of BCG 6 days after the CWS-EAE induction successfully ameliorated EAE symptoms, suggesting the therapeutic effects of BCG is independent of the mycobacterial antigen-specific T cells induced by CFA-EAE protocol. With the CWS-EAE model, we confirmed that induction of MOG-specific Th17 in the spleen and central nervous system (CNS) decreased with disappearance of demyelination lesions by the BCG inoculation. The amelioration of CNS pathology was not linked to changes in the number of macrophages, neutrophil and conventional dendritic cells (DC) but associated with decrease of plasmacytoid DC in CNS. The results suggest that BCG inoculation suppress both systemic and CNS Th17 response in the EAE mice and the mechanism may involve modulation of plasmacytoid DC.
\end{abstract}

Title: A new evidence of therapeutic effects of Mycobacterium bovis BCG vaccine to experimental autoimmune encephalomyelitis (EAE): BCG-mediated suppression of Th17 response in EAE mice induced using an adjuvant without mycobacterial antigen.

Short title: BCG suppressed EAE induced without CFA

Authors: Goro Matsuzaki ${ }^{*}+$, Naoko Teruya* ${ }^{*}$ Hideyasu Kohama Kiyohara, Keiko Arai, Yukihiro Shibuya, Yasushi Chuma, Kazuhiro Matsuo.

Affiliations: *Molecular Microbiology Group, Tropical Biosphere Research Center, and ${ }^{+}$Department of Host Defense, Graduate School of Medicine, University of the Ryukyus, Nishihara , Okinawa 903-0213, Japan.

- = Research and Development Department, Japan BCG Laboratory, Kiyose, Tokyo 204-0022, Japan. 
Correspondence: Goro Matsuzaki, Molecular Microbiology Group, Tropical Biosphere Research Center, University of the Ryukyus, Nishihara, Okinawa 903-0213, Japan.

E-mail: matsuzak@comb.u-rukuy.ac.jp

Key words: EAE, BCG, adjuvant, mycobacterial antigen, Th17

Abbreviations: ANOVA, one-way analysis of variance; BCG,Mycobacterium bovis bacille de Calmette et Guérin; cDC, conventional dendritic cells; CFA, complete Freund's adjuvant; CNS, central nervous system; CWS, cell wall skeleton; EAE, experimental autoimmune encephalomyelitis; ELISA, enzyme-linked immunosorbent assay; FCM, flow cytometry; HE, hematoxylin and eosin; IFA, incomplete Freund's adjuvant; i.p., intraperitoneal; MOG, myelin oligodendrocyte glycoprotein; MS, multiple sclerosis; Mtb, Mycobacterium tuberculosis ; pDC, plasmacytoid dendritic cells; PPD, purified protein derivative of Mtb; s.c., subcutaneous; tSNE, t-distributed stochastic neighbor embedding

\section{Summary}

Multiple sclerosis (MS) is an autoimmune disease mediated by myelin autoantigen-specific T cells. Experimental autoimmune encephalomyelitis (EAE) induced by immunization of mice with a myelin oligodendrocyte glycoprotein (MOG) peptide emulsified in killed Mycobacterium tuberculosis -containing complete Freund's adjuvant (CFA-EAE) is frequently used as a model of MS. Mycobacterium bovis BCG, a vaccine strain with various biological response modifier activity, has been reported to ameliorate clinical symptoms of the CFA-EAE although precise mechanism has not yet been documented. Since the CFA-EAE uses adjuvant with mycobacterial antigens, it is possible that mycobacterial antigen-specific $\mathrm{T}$ cells induced by CFA and those by therapeutic BCG inoculation recognize same antigens, and the cross-reactivity modulate the EAE. To exclude the influence of the cross-reactivity, we established a modified murine EAE model (CWS-EAE) which does not induce mycobacterial antigen-specific T cells. Inoculation of BCG 6 days after the CWS-EAE induction successfully ameliorated EAE symptoms, suggesting the therapeutic effects of BCG is independent of the mycobacterial antigen-specific T cells induced by CFA-EAE protocol. With the CWS-EAE model, we confirmed that induction of MOG-specific Th17 in the spleen and central nervous system (CNS) decreased with disappearance of demyelination lesions by the BCG inoculation. The amelioration of CNS pathology was not linked to changes in the number of macrophages, neutrophil and conventional dendritic cells (DC) but associated with decrease of plasmacytoid DC in CNS. The results suggest that BCG inoculation suppress both systemic and CNS Th17 response in the EAE mice and the mechanism may involve modulation of plasmacytoid DC.

\section{Introduction}

Mycobacterium bovis bacille de Calmette et Guérin (BCG) is a vaccine strain used not only to prevent tuberculosis, but to modulate immune responses in several immune-mediated diseases (1). Clinical trials of BCG therapy against $\mathrm{T}$ cell-mediated autoimmune diseases including type I diabetes and multiple sclerosis (MS) has been reported with promising results (2-4). To establish more effective protocol of the BCG therapy, it is important to elucidate cellular and molecular mechanism of the BCG therapy. To this end, analyses in animal models of the autoimmune diseases are useful. Mouse experimental autoimmune encephalomyelitis (EAE) model is frequently used as an animal model of MS. As a standard EAE model, C57BL/6 mice are immunized with myelin oligodendrocyte glycoprotein (MOG) 35-55 peptide emulsified in complete Freund's adjuvant (CFA) which contains killed Mycobacterium tuberculosis (Mtb) (CFA-EAE mice) (5). This protocol induces MOG-specific Th17 cell-dependent central nervous system (CNS) inflammation and demyelination $(6,7)$.

BCG is used worldwide as a vaccine against tuberculosis because antigenicity of Mtb is extensively shared with that of BCG (8). It imply that Mtb in CFA activates mycobacterial Ag-specific T cells which crossreactive to BCG (9). Although BCG inoculation has been reported to ameliorate symptoms of CFA-EAE mice (10-13), therapeutic effects of BCG on the CFA-EAE mice could be attributed to T cell cross-reactivity between Mtb in CFA and therapeutically inoculated BCG. To rule out influence of such cross-reactivity, an 
EAE model induced without $\mathrm{CFA}$ is required.

In the present report, we established an EAE model induced with cell wall skeleton (CWS). CWS is a protein antigen-deprived cell wall extract of BCG cell wall (14) with strong stimulant activity to innate immunity (15). The mice immunized with MOG peptide plus CWS emulsified in incomplete Freund's adjuvant (IFA) (CWS-EAE mice) showed EAE symptoms with MOG-specific Th17 cells, but no T cell response to mycobacterial antigens. BCG inoculation into the CWS-EAE mice 6 days after the induction resulted in ameliorated clinical symptoms, and reduced Th17 cells in the spleen and CNS. Analysis of non-lymphoid cells infiltrated into the CNS of the CWS-EAE mice showed decrease of plasmacytoid dendritic cells (pDC) which have been reported to regulate EAE. Implications of the observations are discussed.

\section{Materials and methods}

\section{Animals}

C57BL/6 mice were purchased from Japan SLC (Hamamatsu, Japan). Female mice of 10-15 week-old were used in all the experiments. The animal experiments were done under approval of University of the Ryukyus Animal Experiment Committee.

\section{CWS preparation, CWS-EAE induction and evaluation of clinical symptoms}

CWS was prepared according to the method of Uenishi et al . (14) with some modifications. In brief, $M$. bovis BCG Tokyo 172 (ATCC 35737) was cultured and heat-killed at $801^{\circ} \mathrm{C}$ for 30 minutes. Prewashed BCG was suspended in water, disrupted by Mini DeBEE (BEE International, South Easton, MA) at $35 \mathrm{kpsi}$, and centrifuged at $6,800 \times \mathrm{g}$ for 10 minutes at $25^{\circ} \mathrm{C}$. Subsequently, supernatant was centrifuged at $18,000 \times g$ for 1 hour at $25^{\circ} \mathrm{C}$ to crude CWS pellet. To eliminate proteins, the crude CWS was incubated with pronase at $37^{\circ} \mathrm{C}$ for 4 hours in $10 \mathrm{mM}$ Tris- $\mathrm{HCl}$ buffer ( $\mathrm{pH} 8.0$ ) containing $5 \%$ 2-propanol and centrifuged to yield pellets. The pellets was washed with 2-propanol then dried in vacuo to obtain CWS.

CWS-EAE was induced in the C57BL/6 mice by the following method: $2 \mathrm{mg} / \mathrm{ml}$ of $\mathrm{MOG}_{35-55}$ peptide (MBL, Nagoya, Japan) and $1 \mathrm{mg} / \mathrm{ml}$ of CWS emulsified in IFA (BD, San Jose, CA) was administered subcutaneously at upper and lower back with $0.1 \mathrm{ml} /$ site. Two hours and 24 hours after the subcutaneous injection, $400 \mathrm{ng}$ of pertussis toxin (BioAcademia, Osaka, Japan) was intraperitoneally (i.p.) injected. Some groups of mice were also i.p. inoculated with $2 \mathrm{X} 10^{7}$ CFU BCG Tokyo 172 strain (Japan BCG Laboratory, Tokyo, Japan) suspended in PBS at the indicated time point.

Mice were monitored for clinical symptoms and scored 0 to 5 as follows: 0 , no obvious changes in motor function. 0.5, tip of tail is limp. 1.0, limp tail. 1.5, limp tail and hind leg inhibition. 2.0, limp tail and weakness of hind legs. 2.5, limp tail and dragging of hind legs. 3.0, complete paralysis of hind legs or paralysis of one front and one hind leg. 3.5, complete paralysis of hind legs with movement around the cage, or hind legs together on one side of the body. 4.0, complete hind leg and partial front leg paralysis. 4.5, complete hind and partial front leg paralysis, with no movement around the cage. 5.0, spontaneous rolling in the cage, or dead due to paralysis. Mice were euthanized at score 5.

\section{Cell preparation and culture}

Spleen and lymph node cell suspensions were prepared by squeezing the organs between two glass slides. When lymph node cells are used, inguinal and axillar lymph nodes were pooled. To enrich $\mathrm{CD} 4^{+} \mathrm{T}$ cells from the spleen cells, cells were stained with FITC-conjugated anti-CD4 mAb (BioLegend, San Diego, CA), followed by anti-FITC microbeads (Miltenyi Biotech, Bergisch Gladbach, Germany). CD $4^{+}$cells were sorted using AutoMACS cell sorter (Miltenyi Biotech).

To prepare bone marrow-derived macrophages (BMDM), bone marrow cells were collected from the femurs and tibias of mice, cultured with RPMI-1640 medium containing 10\% FBS and 30\% L-929 culture supernatant at $2.5 \times 10^{6}$ cells $/ \mathrm{ml}$ for 7 days with changing medium every 2-3 days. The BMDM were collected, suspended in RPMI1640 medium supplemented with 10\% FBS and antibiotics (penicillin G and strepto- 
mycin), and cultured at $5 \times 10^{5}$ cells $/ \mathrm{ml}$ in 24 well plate with or without $10 \mu \mathrm{g} / \mathrm{ml}$ CWS or BCG for 16 hours.

To prepare CNS-infiltrating cells, CNS were minced using GentleMACS Dissociator (Miltenyi Biotech), incubated with $125 \mathrm{U} / \mathrm{ml}$ collagenase I (ThermoFisher Scentific, Carlsbad, CA) and $60 \mathrm{U} / \mathrm{ml}$ DNase I (Wako Pure Chemical, Osaka, Japan) for 30 minutes at $37^{\circ} \mathrm{C}$, and pass through a stainless steel mesh. The cells were suspended in $37 \%$ Percoll, and centrifuge at $800 \times g$ for 20 minutes at room temperature. The pellets were used as CNS-infiltrating cells.

For in vitro antigen stimulation of T cells, $2.5 \times 10^{5}$ cells were cultured with $10 \mu \mathrm{g} / \mathrm{ml}$ of MOG peptide or 5 $\mu \mathrm{g} / \mathrm{ml}$ of purified protein derivative (PPD) from Mtb (Japan BCG Laboratory) in $200 \mu \mathrm{l}$ RPMI1640 medium supplemented with 10\% FBS and antibiotics (penicillin G and streptomycin) with adherent spleen cells as antigen presenting cells for 48 hours.

\section{Flow cytometry (FCM)}

Cells were stained with mAb specific to leukocyte surface markers, and analyzed with FACSCANTO flow cytometer (BD). In some experiments, cells were stimulated with $10 \mu \mathrm{g} / \mathrm{ml} \mathrm{MOG}_{35-55}$ peptide in the presence of spleen adherent cells as antigen presenting cells for 20 hours. GolgiPlug was then added, incubated for 3 hours, then cells were collected, stained with mAbs against leukocyte surface antigens, fixed, permeabilized and stained for intracellular cytokines using Cytofix/Cytoperm kit (BD) according to the manufacturer's instructions. The antibodies used were Alexa Fluor 700-conjugsted anti-CD4 (BioLegend, San Diego, CA), APC-conjugated anti-CD4 (eBiosceince, San Diego, CA), Alexa Fluor 647-conjugated antiCD11b (BioLegend), biotin-conjugated anti-CD11c (BD), APC/Cy7-conjugated anti-CD45.2 (BioLegend), PE/Dazzle 594-conjugated anti-CD45/B220 (BioLegend), PE-conjugated anti-TCR $\beta$ (BioLegend), biotinconjugated anti-TCR $\beta$ (BD), PerCP/Cy5-conjugated anti-TCR $\gamma \delta$ (BioLegend), FITC-conjugated anti-Gr1 (eBioscience), PerCP/Cy5-conjugated anti-PDCA1 (BioLegend), PE-conjugated anti-IL-17A (BD), Alexa Fluor 700-conjugated anti-IFN- $\gamma$ (BioLegend), and FITC-conjugated anti-IL-10 (BioLegend) mAb. For biotin-conjugated mAb, PE/Dazzle 594-, PE/Cy7- or APC/Cy7-conjugated streptavidin (BioLegend) were used as secondary reagents. The data of FCM was analyzed using FACS DIVA software (BD).

To analyze multiple non-lymphoid cell populations in the CNS, FCM data of CD $45^{+}$cells (four form the EAE mice, and four from the EAE plus BCG mice) was concatenated, then dimensionality of the data was reduced by t-distributed stochastic neighbor embedding (tSNE) algorithm (16) using FlowJo software (BD) . To visualize leukocyte populations in two-dimensional tSNE plots, manually-gated populations of known phenotype were overlaid onto the plots.

\section{Histological analysis}

Spinal cord of mice fixed by perfusion of $4 \%$ paraformaldehyde were embedded in paraffin. Serial paraffin sections were stained with hematoxylin and eosin (HE) to visualize inflammatory cell infiltration and with Luxol fast blue to visualize demyelinated lesions.

\section{Enzyme-linked immunosorbent assay (ELISA)}

IL-1 $\beta$, IL-10, IL-17A, IL-12/23 p40, IFN- $\gamma$ and TNF in culture supernatants were titrated with ELISA method using DuoSet kits (R\&D, Minneapolis, MN) according to the manufacturer's instructions. IL-1b

\section{Statistical analysis}

To compare data of two groups, Student's $t$ test was applied. To compare more than three groups, one-way analysis of variance (ANOVA) was performed followed by Tukey-Kramer multiple comparison test. All the statistical analyses were carried out using the GraphPad InStat software (GraphPad Software, La Jolla, CA). A $P$ value of $<0.05$ was considered to indicate a significant difference.

\section{Results}

Induction of EAE with an adjuvant that do not activate mycobacterial Ag-specific T cells. 
To establish an EAE system induced without mycobacterial antigen-containing adjuvant, we analyzed possibility to use CWS as an adjuvant to induce EAE since CWS has been reported to activate conventional dendritic cells (cDC) via stimulation of TLR (15). As shown in Fig. 1A, CWS induced production of proinflammatory cytokines TNF- $\alpha$ and IL- $1 \beta$ as BCG did. Furthermore, IL-12/23 p40, which is required for induction of Th17and Th1 immunity, is induced by CWS at much higher level compared to that induced by BCG. An anti-inflammatory cytokine IL-10 was also induced by CWS. These data suggest that CWS could be served as Th17 or Th1-inducing adjuvant with IL-10 mediated suppression of excess inflammatory response.

We next immunized mice with MOG peptide emulsified in CWS-containing IFA. As shown in Fig. 1B, the MOG+CWS immunization induced EAE and the incidence was 100\% (Table 1). The EAE induced with CWS is referred as the "CWS-EAE" hereafter. Lymph node cells from the CWS-EAE mice produced IL-17A but not IFN- $\gamma$ in response to MOG peptide while they produced neither IL-17A nor IFN- $\gamma$ in response to mycobacterial PPD (Fig 1C). These results demonstrated that the CWS adjuvant successfully induced EAE without induction of mycobacterial Ag-specific T cell response.

Suppression of EAE symptoms by BCG treatment of mice before or after CWS-EAE induction

To analyze whether BCG suppresses EAE induced in the absence of mycobacterial antigen-containing adjuvant, BCG was i.p. inoculated into the CWS-EAE mice two weeks before or two days after the immunization (Fig. 2A). BCG inoculation two weeks before CWS-EAE induction resulted in significant decrease of clinical score from day 14 to day 19 after the induction (Fig 2B) although there was no significant difference in incidence and maximal clinical score (Table 1). When BCG was inoculated two days after the CWS-EAE induction, not only clinical score but also disease incidence and maximal clinical score decreased (Fig 2C and Table 1). These results suggest that BCG treatment suppress EAE even in the absence of immunization with mycobacterial antigen-containing adjuvant which activate mycobacterial Ag-specific $\mathrm{T}$ cells.

Since treatment of autoimmune diseases would be started after onset of autoimmune response, we next asked whether BCG inoculation effectively suppresses the CWS-EAE model on day 6 of EAE induction when autoreactive Th17 response is establishing (Fig 3A). Although 100\% incidence was observer with or without the late BCG i.p. inoculation, the BCG-inoculated group showed lower clinical score from day 10 of EAE induction (Fig 3B), lower maximal clinical score (Fig. 3C), and delayed disease onset (Fig. 3D). Histological analysis demonstrated demyelinated lesions with inflammatory cell infiltration in the CWS-EAE mice while BCG inoculation into the CWS-EAE mice resulted in normal appearance of the lumber spinal cord (Fig 4). All the results indicate that the BCG inoculation effectively suppresses EAE even at late stage of MOG-specific Th17 induction.

MOG-specific $\mathrm{T}$ cell response in peripheral lymphoid organ modified by the BCG inoculation into the CWS-EAE mice.

To analyze the mechanism of the late BCG inoculation-mediated suppression of the EAE, MOG-specific Th17 cell response was analyzed using spleen $\mathrm{CD} 4^{+} \mathrm{T}$ cells on day 28 of the CWS-EAE induction. The ratio of $\mathrm{IL}_{-17 \mathrm{~A}^{+}}$cells in the spleen $\mathrm{CD} 4^{+} \mathrm{T}$ cells significantly decreased while the ratio of IFN- $\gamma^{+}$cells was not changed by the BCG inoculation on day 6 after the CWS-EAE induction (Fig 5A). ELISA analysis of the culture supernatants of the spleen $\mathrm{CD}^{+} \mathrm{T}$ cells also showed that secretion of IL-17A but not IFN- $\gamma$ significant decreased when BCG was inoculated. Since BCG was inoculated on day 6 after the CWS-EAE induction when MOG-specific Th17 cell development would be already started, BCG may suppress expansion and/or maintenance of MOG-specific Th17 cells.

\section{CNS-infiltrated T cells modified by the BCG inoculation into the CWS-EAE mice.}

To further analyze mechanism of suppression of EAE by the late BCG inoculation, we next analyzed phenotype of $\mathrm{T}$ cells infiltrated in the CNS of the CWS-EAE mice. As shown in Fig 6A, the ratio of TCR $\alpha \beta^{+} \mathrm{CD} 4^{+}$ $\mathrm{T}$ cells significantly decreased by the BCG inoculation into the CWS-EAE mice while the ratio of TCR $\delta^{+}$ 
T cells was not significant different between the two groups. When the infiltrated cells were stimulated with MOG peptide and cytokine production was analyzed by intracellular staining, the BCG inoculation resulted in significant decrease of IL-17A ${ }^{+} \mathrm{CD} 4^{+} \mathrm{T}$ cell population while IFN- $\gamma^{+} \mathrm{CD} 4^{+}$cells showed no significant change (Fig 6B). ELISA analysis of supernatants from the MOG-stimulated CNS-infiltrated cell culture also showed that secretion of IL-17A but not that of IFN- $\gamma$ significant decrease by the BCG inoculation (Fig 6C). All the results demonstrated that the late BCG inoculation into the CWS-EAE mice resulted in decrease of MOG-specific Th17 cells in the CNS, which may be a cause of decreased clinical score in the mice.

\section{CNS-infiltrated non-lymphoid cells modified by the BCG inoculation into the CWS-EAE mice.}

Non-lymphoid cells in the CNS have pivotal role in development of EAE as effector or regulator cells. We next compared CNS-infiltrated non-lymphoid cell populations in the CWS-EAE mice with or without BCG inoculation on day 6 of the EAE induction. To visualize multiple non-lymphoid cell lineage, t-SNE plot of FCM data are shown in Fig 7A. Unexpectedly the ratio of two important effector cells, macrophages $\left(\mathrm{CD} 11 \mathrm{~b}^{+} \mathrm{CD} 11 \mathrm{c}^{\text {low }} \mathrm{Gr} 1^{\text {low }}\right.$ cells) and neutrophils $\left(\mathrm{CD} 11 \mathrm{~b}^{+} \mathrm{Gr} 1^{\text {high }}\right.$ cells), are not different between the two groups of mice. $\mathrm{cDC}\left(\mathrm{CD} 11 \mathrm{~b}^{+} \mathrm{CD} 11 \mathrm{c}^{\text {high }} \mathrm{Gr} 1^{\text {low }}\right.$ cells) which are important in antigen presentation to $\mathrm{T}$ cells were also at similar level between the two group of mice. Interestingly, pDC (PDCA1 ${ }^{+} \mathrm{CD} 11 c^{\text {low }}$ cells) were significantly decreased by the BCG inoculation into the CWS-EAE mice (Fig 7B,C). The phenotype of the pDC were further confirmed to be $\mathrm{CD} 11 \mathrm{~b}^{\text {low }} \mathrm{B} 220^{+}$(Fig 7B) which is consistent with previously reported pDC phenotype (17). pDC have been reported to regulate EAE in suppressive $(13,18-20)$ or enhancing manner (21), and the BCG inoculation would cause suppression of the EAE via reduction of EAE-enhancing function of $\mathrm{pDC}$.

\section{Discussion}

In the present report, we demonstrated therapeutic effects of BCG on murine autoimmune encephalomyelitis using an EAE model induced with an adjuvant free of Mtb which share antigenicity with BCG. It has been reported that BCG therapy ameliorate human MS (1-4), and the mechanism has been analyzed using the CFA-EAE mouse model induced with Mtb-containing adjuvant (10-13). Since BCG share antigenicity with Mtb, effects of the BCG inoculation on the CFA-EAE model could be influenced by the Mtb/BCG-crossreactive $\mathrm{T}$ cells. Inoculation of other mycobacterial preparations including killed Mtb, killed Mtb-containing CFA, and $12 \mathrm{kD}$ protein of Mtb-derived PPD before the CFA-EAE induction also ameliorated EAE (22-24). In the systems, reactivation of Mtb-specific T cells by CFA could have pivotal effects in suppression of EAE. To rule out influence of the cross-reactivity between BCG and Mtb in CFA during BCG therapy against EAE, an EAE model which do not induce mycobacterial Ag-specific T cells are required. The CWS-EAE model did not induce mycobacterial Ag-specific T cell response, and BCG inoculation before or after the CWS-EAE induction effectively suppressed symptoms of the EAE. These data clearly demonstrates that BCG can suppress EAE irrespective of the presence of Mtb during EAE induction. The CWS-EAE model is therefore a very useful model to analyze mechanisms of BCG-mediated suppression of EAE.

Our analyses with the CWS-EAE model demonstrated that BCG i.p. inoculation on day 6 of EAE induction suppress CNS autoantigen-specific pathogenic Th17 response in both the spleen and CNS. Similar results were obtained using other models. Intracerebral inoculation of BCG 21 days before CFA-EAE induction completely blocked development of encephalomyelitis with decreased MOG-specific Th17 and Th1 responses in the CNS but not in peripheral lymphoid organs (12). Subcutaneous (s.c.) inoculation of BCG inactivated by extended freeze-drying on the day of the CFA-EAE induction resulted in decrease of total Th17 and Th1 in the CNS and spleen (13). These observations suggest that BCG inoculation may suppress development of EAE in two mechanisms. First, BCG suppress development of MOG-specific Th17 in peripheral lymphoid organs which is supported by our experiments and that of the experiments of inactivated BCG s.c. into the CFA-EAE model (13). Second, BCG suppresses migration of the CNS autoantigen-specific Th17 into the CNS when BCG accessed in the CNS. This is demonstrated by intracerebral BCG inoculation to the CFAEAE mice (12). It is possible that i.p. inoculation of BCG not only suppressed induction of MOG-specific Th17 cells, but also inhibited migration of the Th17 cells into CNS after distribution of BCG in the CNS. We need further information on access of BCG or BCG-phagocytosed macrophages into the CNS to clarify 
which mechanism is dominant in the BCG i.p. therapy model of EAE.

In contrast to Th17-mediated EAE model, a Th1-mediated EAE model of Lewis rat failed to show therapeutic effect of BCG. When Lewis rats were immunized with myelin basic protein emulsified in CFA, EAE was induced with Th1 infiltration into the spinal cord (25). In the rat EAE model, BCG inoculation failed to ameliorate EAE symptoms (26). The observation suggests that BCG inoculation is effective to prevent EAE when it depends on Th17 but it may not effective to Th1-dependent EAE. Since various T cell populations including Th17, Th1 and CD8 T cells are involved in MS (27), BCG therapy could be effective only on MS patients with autoantigen-specific Th17 as dominant pathogenic T cells. Study on correlation between phenotype of pathogenic encephalitogenic T cells and efficacy of BCG therapy in MS patients would give useful information to select MS patients with good response to the therapy.

Various lymphoid and non-lymphoid cells in the CNS other than conventional $\mathrm{T}$ cells have been reported to positively regulate murine EAE. Similar to Th17, TCR $\gamma \delta \mathrm{T}$ cells infiltrated in the CNS of EAE mice produce IL-17A and enhance migration of neutrophils and monocyte-deriver macrophages in the CNS to enhance EAE $(28,29)$. Although BCG inoculation suppressed Th17 in the CNS, TCR $\gamma \delta \mathrm{T}$ cells was not influenced by BCG inoculation in our experimental setting. Therefore, TCR $\gamma \delta \mathrm{T}$ cells are not involved in the mechanism of the BCG-mediated suppression of EAE. Macrophages and neutrophils induced by IL-17A into the CNS are also important in pathogenesis of EAE (30,31). Unexpectedly, the non-lymphoid leukocytes were not decreased by BCG therapy to EAE. It is possible that the inflammatory cells in the CNS failed to induce pathogenic demyelination when Th17 response is below optimal level. Further analysis of the immune status in the CNS of the BCG-treated CWS-EAE mice will clarify why inflammatory lesions and demyelination is suppressed in the presence of the inflammatory cells.

$\mathrm{pDC}$ have been reported to have important role in regulation of EAE in both positive and negative manner. Depletion of pDC from day 8 of EAE induction (18), or pDC-selective MHC class II abrogation (19) exacerbated clinical symptoms with enhanced Th17 and Th1 responses. When MOG peptide-loaded pDC were transferred to EAE-induced mice after onset of the disease, the mice showed decreased symptoms (20). These observations support suppressive role of pDC to EAE. In contrast, pDC depletion before EAE induction resulted in suppression of EAE symptoms with reduced Th17 response which seems to be mediated by type I interferon (21). This suggests possibility of pDC-mediated enhancement of EAE. In a BCG therapy model, it was reported that suppression of EAE by inactivated BCG was cancelled by pDC depletion, and transfer of pDC derived from bone marrow of inactivated BCG-treated mice suppressed EAE (13). From the observation, it is estimated that $\mathrm{pDC}$ participate in suppression of EAE after BCG treatment. Unexpectedly, our observation demonstrates that BCG inoculation resulted in decrease of $\mathrm{pDC}$ in CNS of the CWS-EAE mice. Although the function of CNS-infiltrating $\mathrm{pDC}$ in the CWS-EAE mice is unclear, it is possible that decrease of $\mathrm{pDC}$ resulted in down regulation of pDC-mediated enhancement of Th17 response as observer by Isaksson et al. (21). We need further functional analyses to clarify real regulatory function of CNS pDC against EAE, and the CWS-EAE system would be useful in the analysis.

\section{Acknowledgements}

Authors' contributions: G.M., H.K.K. and K.M. designed research. G.M. wrote the paper. G.M. and N.T. performed animal and cell experiments. H.K.K., K.A., Y.S., and Y.C. purified and prepared materials used in the experiments.

All the authors read and approved the final manuscript.

\section{Disclosure}

The authors have no financial conflicts of interest.

\section{References}

1. Ristori G, Faustman D, Matarese G, Romano S, Salvetti M. Bridging the gap between vaccination with Bacille Calmette-Guérin (BCG) and immunological tolerance: the cases of type 1 diabetes and multiple sclero- 
sis. Curr Opin Immunol [Internet]. 2018;55:89-96. Available from: https://doi.org/10.1016/j.coi.2018.09.016

2. Ristori G, Buzzi MG, Sabatini U, Giugni E, Bastianello S, Viselli F, et al. Use of Bacille Calmette-Guerin (BCG) in multiple sclerosis. Neurology. 1999;53(7):1588-9.

3. Paolillo A, Buzzi MG, Giugni E, Sabatini U, Bastianello S, Pozzilli C, et al. The effect of Bacille CalmetteGuérin on the evolution of new enhancing lesions to hypointense T1 lesions in relapsing remitting MS. J Neurol. 2003;250(2):247-8.

4. Ristori G, Romano S, Cannoni S, Visconti A, Tinelli E, Mendozzi L, et al. Effects of Bacille CalmetteGuérin after the first demyelinating event in the CNS. Neurology. 2014;82(1):41-8.

5. Mendel I, de Rosbo NK, Ben-Nun A. A myelin oligodendrocyte glycoprotein peptide induces typical chronic experimental autoimmune encephalomyelitis in $\mathrm{H}-2 \mathrm{~b}$ mice: Fine specificity and $\mathrm{T}$ cell receptor V $\beta$ expression of encephalitogenic T cells. Eur J Immunol. 1995;25(7):1951-9.

6. Cua DJ, Sherlock J, Chen Y, Murphy CA, Joyce B, Seymour B, et al. Interleukin-23 rather than interleukin-12 is the critical cytokine for autoimmune inflammation of the brain. Nature. 2003;421(6924):744-8.

7. Langrish CL, Chen Y, Blumenschein WM, Mattson J, Basham B, Sedgwick JD, et al. IL-23 drives a pathogenic T cell population that induces autoimmune inflammation. J Exp Med. 2005;201(2):233-40.

8. Zimpel CK, Brandão PE, de Souza Filho AF, de Souza RF, Ikuta CY, Neto JSF, et al. Complete genome sequencing of Mycobacterium bovis SP38 and comparative genomics of Mycobacterium bovis and M. tuberculosis strains. Front Microbiol. 2017;8(DEC):1-14.

9. Billiau A, Matthys P. Modes of action of Freund's adjuvants in experimental models of autoimmune diseases. J Leukoc Biol [Internet]. 2001;70(6):849-60. Available from: http://www.ncbi.nlm.nih.gov/pubmed/11739546

10. Sewell D, Reinke E, Co D, Hogan L, Fritz R, Sandor M, et al. Infection with Mycobacterium bovis BCG Diverts Traffic of Myelin Oligodendroglial Glycoprotein Autoantigen-Specific T Cells Away from the Central Nervous System and Ameliorates Experimental Autoimmune Encephalomyelitis. Clin Diagn Lab Immunol. 2003;10(4)(May 2011):564-572.

11. O'Connor RA, Wittmer S, Dalton DK. Infection-induced apoptosis deletes bystander CD4+ T cells: A mechanism for suppression of autoimmunity during BCG infection. J Autoimmun. 2005;24(2):93-100.

12. Lee J, Reinke EK, Zozulya AL, Sandor M, Fabry Z. Mycobacterium bovis Bacille Calmette-Guérin Infection in the CNS Suppresses Experimental Autoimmune Encephalomyelitis and Th17 Responses in an IFN- $\gamma$-Independent Manner . J Immunol. 2008;181(9):6201-12.

13. Lippens C, Garnier L, Guyonvarc'h PM, Santiago-Raber ML, Hugues S. Extended freeze-dried BCG instructed pDCs induce suppressive tregs and dampen EAE. Front Immunol. 2018;9(NOV):1-12.

14. Uenishi Y, Okada T, Okabe S, Sunagawa M. Study on the cell wall skeleton derived from Mycobacterium bovis BCG Tokyo 172 (SMP-105): Establishment of preparation and analytical methods. Chem Pharm Bull. 2007;55(6):843-52.

15. Tsuji S, Matsumoto M, Takeuchi O, Akira S, Azuma I, Hayashi A, et al. Maturation of human dendritic cells by cell wall skeleton of Mycobacterium boris bacillus Calmette-Guérin: Involvement of toll-like receptors. Infect Immun. 2000;68(12):6883-90.

16. Maaten L van der, Hinton G. Visualizing Data using t-SNE Laurens. J Mach Learn Res. 2008;9:2579-605.

17. Swiecki M, Colonna M. The multifaceted biology of plasmacytoid dendritic cells. Nat Rev Immunol. 2015;15(8):471-85. 
18. Bailey-Bucktrout SL, Caulkins SC, Goings G, Fischer JAA, Dzionek A, Miller SD. Cutting Edge: Central Nervous System Plasmacytoid Dendritic Cells Regulate the Severity of Relapsing Experimental Autoimmune Encephalomyelitis. J Immunol. 2008;180(10):6457-61.

19. Irla M, Küpfer N, Suter T, Lissilaa R, Benkhoucha M, Skupsky J, et al. MHC class II-restricted antigen presentation by plasmacytoid dendritic cells inhibits $\mathrm{T}$ cell-mediated autoimmunity. J Exp Med. 2010;207(9):1891-905.

20. Duraes F V., Lippens C, Steinbach K, Dubrot J, Brighouse D, Bendriss-Vermare N, et al. PDC therapy induces recovery from EAE by recruiting endogenous pDC to sites of CNS inflammation. J Autoimmun [Internet]. 2016;67:8-18. Available from: http://dx.doi.org/10.1016/j.jaut.2015.08.014

21. Isaksson M, Ardesjö B, Rönnblom L, Kämpe O, Lassmann H, Eloranta ML, et al. Plasmacytoid DC promote priming of autoimmune Th17 cells and EAE. Eur J Immunol. 2009;39(10):2925-35.

22. Lehmann D, Ben-Nun A. Bacterial agents protect against autoimmune disease. I. Mice pre-exposed to Bordetella pertussis or Mycobacterium tuberculosis are highly refractory to induction of experimental autoimmune encephalomyelitis. J Autoimmun. 1992;5(6):675-90.

23. O'Connor RA, Li X, Blumerman S, Anderton SM, Noelle RJ, Dalton DK. Adjuvant Immunotherapy of Experimental Autoimmune Encephalomyelitis: Immature Myeloid Cells Expressing CXCL10 and CXCL16 Attract CXCR3 + CXCR6 + and Myelin-Specific T Cells to the Draining Lymph Nodes Rather Than the Central Nervous System. J Immunol. 2012;188(5):2093-101.

24. Ben-Nun A, Mendel I, Sappler G, Rosobo N de. A 12-kDa protein of Mycobacterium tuberculosis protect mice atgainst experimental autoimmune encephalomyelitis. Protection in the absence of shared $\mathrm{T}$ cell epitopes with encephalitogenic proteins. J Immunol. 1995;154:2939-48.

25. Almolda B, Costa M, Montoya M, González B, Castellano B. Increase in th17 and t-reg lymphocytes and decrease of il22 correlate with the recovery phase of acute eae in rat. PLoS One. 2011;6(11):1-13.

26. Zorzella-Pezavento SFG, Guerino CPF, Chiuso-Minicucci F, França TGD, Ishikawa LLW, Masson AP, et al. BCG and BCG/DNAhsp65 vaccinations promote protective effects without deleterious consequences for experimental autoimmune encephalomyelitis. Clin Dev Immunol. 2013;2013.

27. Fletcher JM, Lalor SJ, Sweeney CM, Tubridy N, Mills KHG. T cells in multiple sclerosis and experimental autoimmune encephalomyelitis. Clin Exp Immunol. 2010;162(1):1-11.

28. Sutton CE, Lalor SJ, Sweeney CM, Brereton CF, Lavelle EC, Mills KHG. Interleukin-1 and IL-23 Induce Innate IL-17 Production from $\gamma \delta$ T Cells, Amplifying Th17 Responses and Autoimmunity. Immunity [Internet]. 2009;31(2):331-41. Available from: http://dx.doi.org/10.1016/j.immuni.2009.08.001

29. Petermann F, Rothhammer V, Claussen MC, Haas JD, Blanco LR, Heink S, et al. $\gamma \delta$ T Cells Enhance Autoimmunity by Restraining Regulatory T Cell Responses via an Interleukin-23-Dependent Mechanism. Immunity [Internet]. 2010;33(3):351-63. Available from: http://dx.doi.org/10.1016/j.immuni.2010.08.013

30. Lévesque SA, Paré A, Mailhot B, Bellver-Landete V, Kébir H, Lécuyer MA, et al. Myeloid cell transmigration across the CNS vasculature triggers IL-1ß-driven neuroinflammation during autoimmune encephalomyelitis in mice. J Exp Med. 2016;213(6):929-49.

31. McGinley AM, Sutton CE, Edwards SC, Leane CM, DeCourcey J, Teijeiro A, et al. Interleukin17A Serves a Priming Role in Autoimmunity by Recruiting IL-1 $\beta$-Producing Myeloid Cells that Promote Pathogenic T Cells. Immunity [Internet]. 2020;52(2):342-356.e6. Available from: https://doi.org/10.1016/j.immuni.2020.01.002

\section{Figure legends}

Fig. 1 Induction of EAE by MOG immunization with CWS as a mycobacterial antigen-free adjuvant. A) BMDM were cultured with or without $10 \mu \mathrm{g} / \mathrm{ml}$ of CWS or BCG, and cytokines in the supernatants were 
titrated by ELISA. A representative data of two independent experiments is shown. ${ }^{*} P<0.05$ in one way ANOVA and Tukey-Kramer multiple comparisons test. B) C57BL/6 mice were s.c. inoculated with MOG peptide emulsified with IFA and CWS and clinical symptoms of the CWS-EAE mice was monitored as described in Materials and Methods. The experiments were repeated more than five times and all the mice used showed clinical symptoms. The data shown is a representative data with 10 mice. C) Lymph node cell suspensions were prepared on day 28 of the CWS-EAE induction, cultured in the presence or absence of mycobacterial antigen (PPD) or MOG peptide, and cytokines in the supernatants were titrated by ELISA. A representative data of two independent experiments are shown. ${ }^{*} P<0.05$ in one way ANOVA and Tukey-Kramer multiple comparisons test.

Fig. 2 BCG inoculation ameliorates clinical symptoms of the CWS-EAE mice. A) A schematic representation of experimental protocol. B,C) BCG inoculation 14 days before (B) or 2 days after (C) the CWS-EAE induction suppressed clinical symptoms of EAE. ${ }^{*} P<0.05,{ }^{\#} P<0.005$ compared to the CWS-EAE mice without BCG inoculation in Student's $t$ test.

Fig.3 BCG inoculation six days after the CWS-EAE induction ameliorated symptoms of the EAE. A) A schematic representation of experimental protocol. B) BCG inoculation 6 days after the CWS-EAE induction suppressed clinical symptoms of EAE. The data is a representative of three independent experiments. ${ }^{*} P$ $<0.05,{ }^{\#} P<0.005$ compared to the CWS-EAE mice without BCG inoculation in Student'st test. C,D) Maximal clinical score (C) and onset of disease (D) were compared between the CWS-EAE mice and those with BCG inoculation on day 6 of the induction. Statistical analysis was carried out with Student's $t$ test.

Fig. 4 BCG inoculation ameliorated demyelinated lesions of CWS-EAE mice. The CWS-EAE mouse (middle panels) showed inflammatory cell infiltration (HE staining) with demyelination lesions (arrowheads in Luxol blue staining) while the CWS-EAE mouse with BCG inoculation on day 6 (lower panels) showed no inflammatory or demyelinated lesion as the normal spinal cord (upper panels).

Fig. 5 BCG inoculation suppressed MOG-specific Th17 response in the spleen of the CWS-EAE mice. Spleen cells were prepared on day 28 of the induction, enriched for CD4 ${ }^{+}$cells, and stimulated with MOG peptide in the presence of normal spleen adherent cells as antigen presenting cell. A) Production of IL$17 \mathrm{~A}$ and $\mathrm{IFN}-\gamma$ by $\mathrm{CD}^{+} \mathrm{T}$ cells were analyzed by FCM. A representative contour plots are shown and summarized in bar graphs. B) Culture supernatants were analyzed by ELISA for their contents of IL-17A and IFN- $\gamma$. Statistical analysis was carried out with Student's ttest.

Fig. 6 BCG inoculation suppressed MOG-specific Th17 response in the CNS of the CWS-EAE mice. CNSinfiltrated cells were prepared on day 28 of the CWS-EAE induction with or without BCG inoculation on day 6. A) TCR and CD4 expression of the freshly isolated CNS-infiltrated cells were analyzed by FCM. A representative contour plots are shown and summarized in bar graphs. B) The CNS-infiltrated cells were cultured with spleen antigen presenting cells with MOG peptide, and production of IL-17A and IFN- $\gamma$ by $\mathrm{CD}^{+} \mathrm{T}$ cells were analyzed by FCM. A representative contour plots are shown and summarized in bar graphs. C) Culture supernatant from B was analyzed by ELISA for their contents of IL-17A and IFN- $\gamma$, and calculated to represent cytokine production per total CNS-infiltrated cells. Statistical analysis was carried out with Student's $t$ test.

Fig. 7 BCG inoculation on day 6 of the CWS-EAE induction suppressed accumulation of pDC but did not affect macrophages, neutrophil and $\mathrm{cDC}$ in the CNS of the CWS-EAE mice. A) Multiple parameter FCM data of CNS-infiltrated $\mathrm{CD} 45^{+}$cells are shown in tSNE plot. Colors correspond to manual annotation of non-lymphoid leukocyte populations. Representative profiles of CNS-infiltrated cells from a CWS-EAE mouse and a CWS-EAE mouse with BCG inoculation are shown. The right table indicate phenotypes of analyzed populations and percentages of the populations in the plot. B) FCM profile of pDC identified in the CNS-infiltrated cells as PDCA $1^{+} \mathrm{CD} 11 \mathrm{c}^{\text {low }} \mathrm{CD} 11 \mathrm{~b}^{+} \mathrm{B} 220^{+}$cells. C) Percentage and absolute number of $\mathrm{pDC}$ in the CNS. Statistical analysis was carried out with Student's $t$ test.

\section{Hosted file}


Matsuzaki et al Table 1.xlsx available at https://authorea.com/users/352495/articles/ 476728-a-new-evidence-of-therapeutic-effects-of-mycobacterium-bovis-bcg-vaccine-toexperimental-autoimmune-encephalomyelitis-eae-bcg-mediated-suppression-of-th17-responsein-eae-mice-induced-using-an-adjuvant-without-mycobacterial-antigen

Hosted file

Matsuzaki et al Figs.ppt available at https://authorea.com/users/352495/articles/476728-anew-evidence-of-therapeutic-effects-of-mycobacterium-bovis-bcg-vaccine-to-experimentalautoimmune-encephalomyelitis-eae-bcg-mediated-suppression-of-th17-response-in-eae-miceinduced-using-an-adjuvant-without-mycobacterial-antigen 